\title{
Nanoscale Chemical Imaging of a Single Catalyst Particle with Tip-Enhanced Fluorescence Microscopy
}

\author{
Naresh Kumar, ${ }^{[a, b]}$ Sam Kalirai, ${ }^{[a]}$ Andrew J. Wain, ${ }^{[b]}$ and Bert M. Weckhuysen ${ }^{*[a]}$
}

\begin{abstract}
Determining the active site in real-life solid catalysts remains an intellectual challenge and is crucial for exploring the road towards their rational design. In recent years various microspectroscopic methods have revealed valuable structure-activity data at the level of a single catalyst particle, even under reaction conditions. Herein, we introduce Tip-Enhanced FLuorescence (TEFL) microscopy as a novel and versatile characterization tool for catalysis research. This has been achieved using a Fluid Catalytic Cracking (FCC) catalyst as showcase material. Thin sectioning of industrially used FCC particles together with selective staining of Brønsted acidity has enabled highresolution TEFL mapping of different catalyst regions. Hyperspectral information gained via TEFL microscopy reveals a spatial distribution of Brønsted acidity within individual zeolite domains in different regions of the FCC catalyst particle. Comparison of TEFL measurements from different FCC particles showed significant intra- and inter-particle heterogeneities both in zeolite domain size and chemical reactivity.
\end{abstract}

During the last two decades, significant progress has been made in the use of Localized Surface Plasmon Resonance (LSPR) generated at the apex of a metallic scanning probe under laser excitation as a means to enhance optical signals. ${ }^{[1]}$ The physical principle underlying this approach, broadly referred to as TipEnhanced Optical Spectroscopy (TEOS), is enhancement of the local Electro-Magnetic (EM) field and its confinement to a small volume of space surrounding the probe apex. ${ }^{[2]}$ This near-field phenomenon increases the chemical sensitivity of TEOS and pushes its spatial resolution far beyond the diffraction limit of light. To date, TEOS has been predominantly used for enhanc-

[a] N. Kumar, Dr. S. Kalirai, Prof. Dr. B. M. Weckhuysen

Inorganic Chemistry and Catalysis Group

Debye Institute for Nanomaterials Science

Utrecht University

Universiteitsweg 99

Utrecht 3584 CG (The Netherlands)

E-mail: B.M.Weckhuysen@uu.nl

[b] N. Kumar, Dr. A. J. Wain

National Physical Laboratory

Hampton Road

Teddington, TW11 OLW (United Kingdom)

Supporting information for this article is available on the WWW under https://doi.org/10.1002/cctc.201801023

- This manuscript is part of the Anniversary Issue in celebration of 10 years of ChemCatChem.

of (c) 2018 The Authors. Published by Wiley-VCH Verlag GmbH \& Co. KGaA. This is an open access article under the terms of the Creative Commons Attribution Non-Commercial NoDerivs License, which permits use and distribution in any medium, provided the original work is properly cited, the use is non-commercial and no modifications or adaptations are made. ing two types of optical signal: Raman scattering and photoluminescence emission. Enhancement of Raman signals in this way is commonly known as Tip-Enhanced Raman Spectroscopy (TERS), ${ }^{[3]}$ and has been extensively used for nanoscale chemical characterisation in a wide range of disciplines, including biology ${ }_{r}^{[4]}$ material science, ${ }^{[5]}$ polymer-blends, ${ }^{[6]}$ solar-cells, ${ }^{[7]}$ catalysis $^{[8]}$ single molecule detection, ${ }^{[9]}$ carbon nanotubes ${ }^{[10]}$ and two-dimensional materials such as graphene. ${ }^{[11,12]}$ On the other hand, its counterpart Tip-Enhanced Photo-Luminescence (TEPL) microscopy has remained rather underexplored. ${ }^{[13]}$ This is particularly true in the case of fluorescence microscopy, with only a handful of published reports demonstrating TipEnhanced FLuorescence (TEFL) measurements. ${ }^{[14]}$

In this work, we demonstrate the application of TEFL microscopy for nanoscale investigation of zeolite domains within a Fluid Catalytic Cracking (FCC) particle. FCC is a leading industrial process for the conversion of crude oil fractions into valuable products, such as gasoline and propylene. ${ }^{[15]}$ This catalytic process utilizes $50 \mu \mathrm{m}-150 \mu \mathrm{m}$ sized spherical particles containing a zeolite component, such as zeolite $\mathrm{Y}$ or ZSM-5, embedded in a matrix of clay, silica, and alumina. The cracking properties of the FCC particles are critically determined by the Brønsted acid sites present within the zeolite domains. Cyclical catalysis and regeneration processes of the FCC particles result in a decrease in the strength and number of Brønsted acid sites within the zeolite domains leading to the lowering of their overall catalytic reactivity. However, due to their inherent chemical and structural complexity, a detailed nanoscale characterization of the Brønsted acid sites and their spatial distribution within a FCC particle remains extremely challenging.

Previously, Confocal Fluorescence Microscopy (CFM) has been successfully applied to visualize Brønsted acidity within single FCC particles, differing in their catalytic cracking activity, using various Brønsted acid-catalysed staining reactions. ${ }^{[16]}$ However, due to its diffraction-limited spatial resolution and relatively low sensitivity, CFM cannot provide information about the catalytically active zeolite domains at the nanometre length scale. Recently, Single-Molecule Fluorescence (SMF) microscopy has been successfully utilized for the investigation of zeolite domains within FCC particles. ${ }^{[17]}$ However, SMF is still restricted in resolution for the case of non-dilute fluorescent events and requires relatively complicated data processing. ${ }^{[17]}$ Furthermore, unlike SMF, TEFL provides two-dimensional hyperspectral information, which can allow simultaneous measurement of multiple fluorescent species having different emission wavelengths, enabling detailed chemical information to be gleaned. In other words, TEFL provides, in contrast to SMF and CFM, 
direct chemical information at the nanoscale without the requirement for complex data processing techniques.

Herein, we have applied TEFL to directly map the catalytic activity within industrially applied FCC catalyst particles containing zeolite $\mathrm{Y}$ as the active phase. TEFL microscopy is implemented by placing a Ag-coated Atomic Force Microscopy (AFM) probe within the excitation laser spot of an inverted confocal optical microscope (Figure 1a). We have used the Brønsted acid-catalysed oligomerization of thiophene as a probe molecule, which leads to the formation of fluorescent conjugated carbocationic species of varying length (Figure $1 \mathrm{~b}$ ). An example of a high-resolution fluorescence image obtained using TEFL is shown in Figure 1c. Since the fluorescent species are exclusively formed at the Brønsted acid sites within the zeolite aggregates, their fluorescence emission intensity can be directly used to localize and evaluate Brønsted acidity and hence directly determine the size and inherent reactivity of the zeolite domains. We show that high-resolution spectroscopic information gained via TEFL provides detailed insights into the distribution of Brønsted acidity within individual zeolite domains and reveals significant intra- and inter-particle variations in both their size and reactivity.

We first tested the sensitivity of Ag-coated TEFL probes (Figure 1d) for detection of the fluorescence signal originating from the carbocationic species formed from the oligomerization of the thiophene probe molecule. Figure 1e shows the TEFL and far-field fluorescence spectra measured with a TEFL probe in contact and retracted from a zeolite region within a thin FCC section on a glass substrate (Figure 2a). Two prominent fluorescence bands around $650 \mathrm{~nm}$ and $700 \mathrm{~nm}$ are observed in these spectra, which are assigned to distinct conjugated oligomeric carbocationic species containing $>4$ monomer units



(a)

(Fluorescent)

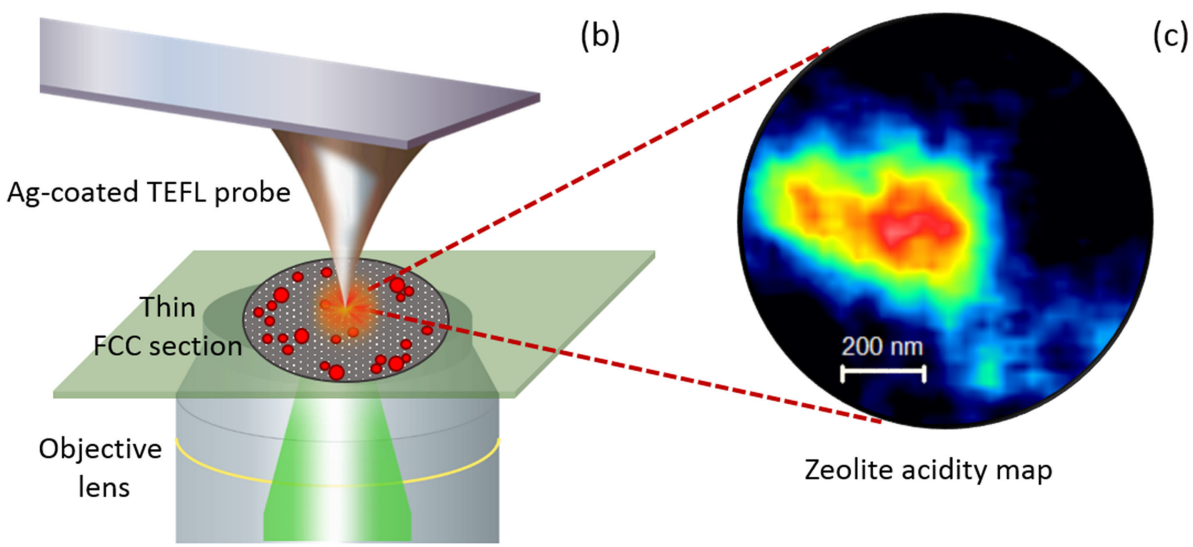

(c)

Excitation laser

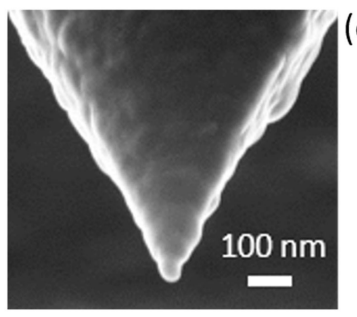

Ag-coated TEFL probe
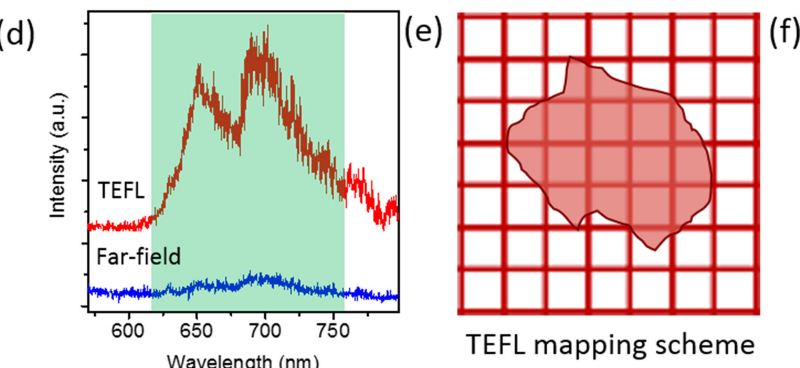

Figure 1. a) Brønsted acid-catalysed oligomerization of thiophene to form fluorescent carbocationic species at the Brønsted acid sites of zeolite domains within an FCC particle. In this study, spherical FCC particles were embedded in an epoxy resin and cut to $100 \mathrm{~nm}$ thickness. Sectioned FCC samples were placed on a glass coverslip and the epoxy resin was removed via calcination. Thin FCC sections were stained by exposure to thiophene and the oligomerisation reaction was carried out by heating the stained FCC sections at $120^{\circ} \mathrm{C}$ for $30 \mathrm{~min}$. FCC sections containing thiophene oligomerisation species at Brønsted acid sites were then analysed using TEFL microscopy. See Supplementary Information (SI) section S1.3 for more details. b) Schematic of the TEFL microscopy configuration used in this work. c) Chemical image of acidity distribution within an individual zeolite domain obtained via TEFL mapping. d) Scanning electron microscopy (SEM) image of a representative Ag-coated TEFL probe used in this work. Typical diameter of the probe-apex is $\approx 50$ nm. e) An exemplary TEFL spectrum (red) measured with the TEFL probe in contact with the FCC sample and the far-field FL spectrum (blue) measured at the same position with the TEFL probe retracted from the FCC sample. Integration time: $10 \mathrm{~s}$. Laser power: $365 \mu \mathrm{W}$. Excitation laser: $532 \mathrm{~nm}$. The scale on the $\mathrm{y}$-axis is in arbitrary units (a.u). f) Diagram illustrating the TEFL mapping scheme. A thin FCC section placed on a glass substrate is moved in a raster fashion between the TEFL probe and excitation laser as shown in Figure 1a whilst measuring a TEFL spectrum at each pixel of the mapped region. Further details are presented in SI section S1. 

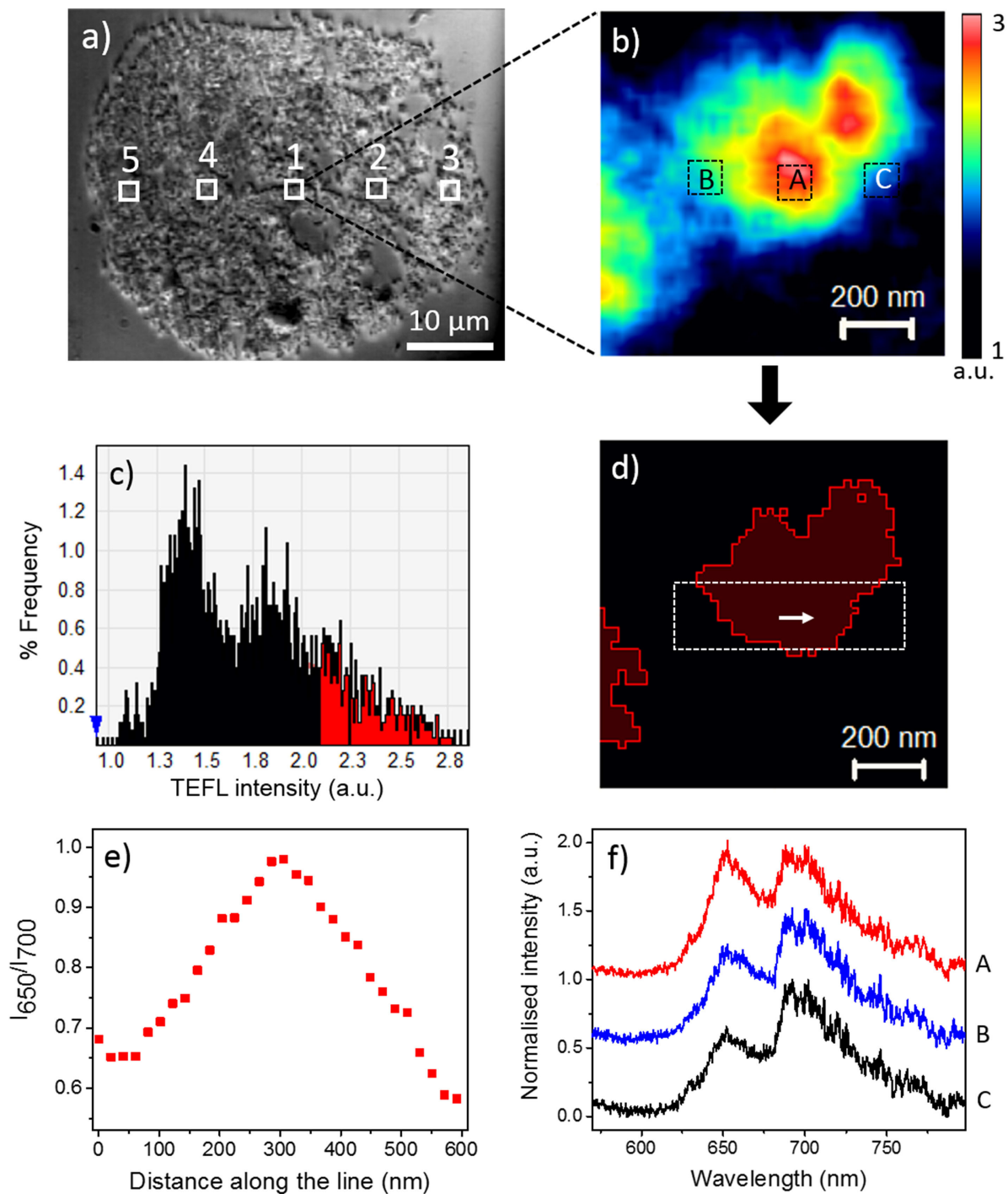

Figure 2. a) Optical image of a thin section (nominal thickness: $100 \mathrm{~nm}$ ) of an industrially spent FCC particle (FCC1) placed on glass substrate. b) TEFL chemical map obtained using the total intensity of the $650 \mathrm{~nm}$ and $700 \mathrm{~nm}$ TEFL bands measured in a $1 \mu \mathrm{m} \times 1 \mu \mathrm{m}$ area marked as 1 in Figure $2 \mathrm{a}$ Nominal pixel size: $20 \mathrm{~nm}$. Integration time: $1 \mathrm{~s}$. Laser power: $365 \mu \mathrm{W}$. c) Histogram of the TEFL intensity $\left(\mathrm{I}_{\mathrm{FL}}\right)$ in the map shown in Figure $2 \mathrm{~b}$ Red region represents TEFL intensity of the catalytically active zeolite domains. d) Binary image of the most catalytically active zeolite regions in the TEFL map shown in Figure $2 \mathrm{~b}$-e) Profile of the average intensity ratio of the $650 \mathrm{~nm}$ and $700 \mathrm{~nm}$ TEFL bands $\left(\mathrm{I}_{650} / \mathrm{I}_{700}\right)$ measured in the region marked in Figure $2 \mathrm{~d}$ along the direction of the arrow. $\left.\mathrm{f}\right)$ Average TEFL spectra from positions A-C marked in Figure $2 \mathrm{~b}$. Spectra have been normalised and vertically shifted for easier visualisation.

formed via the catalytic conversion of thiophene at Brønsted acid sites. ${ }^{[16 d, 18]}$ In the TEFL spectrum, a higher fluorescence intensity is observed due to the plasmonic enhancement of the EM field intensity at the probe-apex. Similar to TERS measurements, ${ }^{[19]}$ the enhancement of a TEFL signal compared to the far-field FL signal can be calculated using "contrast" [Eq. (1)], which is defined as ${ }^{[19]}$

Contrast $=\frac{I_{\text {TEFL }}}{I_{\text {Far }- \text { field }}}-1$

where, $I_{\text {TEFL }}$ and $I_{\text {Far-field }}$ are the intensity of fluorescence signal measured with the TEFL probe in contact and retracted from the sample, respectively. Using Equation (1), the contrast of the $700 \mathrm{~nm}$ band in the TEFL spectrum in Figure 1e is estimated to be 9.3. This shows that the Ag-coated TEFL probe undergoes efficient LSPR with the $532 \mathrm{~nm}$ excitation laser leading to a strong enhancement of the local field intensity at the probeapex. The high EM intensity in the tip-enhanced near-field leads to a strong enhancement of fluorescence signal from the carbocationic thiophene species present beneath the probeapex as discussed in SI section S1.4. To rule out the possibility of $\mathrm{Ag}$ initiating further polymerisation of thiophene, we compared the surface-enhanced Raman spectrum of thiophene measured on a nanostructured Ag substrate with the confocal Raman spectrum (see Figure S4). The measurements confirmed the inertness of $\mathrm{Ag}$ for the oligomerisation of thiophene.

Using plasmonically-active TEFL probes, we carried out high-resolution fluorescence microscopy of thin FCC sections following the scheme illustrated in Figure 1f. This has been carried out for three FCC catalyst particles, labelled as FCC1, FCC2 and FCC3. The hyperspectral TEFL mapping was per- 
formed in a $1 \mu \mathrm{m} \times 1 \mu \mathrm{m}$ area marked as 1 in the centre of a FCC particle, as shown in Figure 2a. TEFL spectra in the $540 \mathrm{~nm}-800 \mathrm{~nm}$ range were collected from $50 \times 50$ pixels $(20 \mathrm{~nm} \times 20 \mathrm{~nm}$ pixel size) in the mapped region. The TEFL spectra measured at each pixel in the map were fitted using two Gaussian curves centred at $650 \mathrm{~nm}$ and $700 \mathrm{~nm}$ after linear background subtraction and the total intensity of the fitted Gaussian curves was used to construct the TEFL map shown in Figure $2 \mathrm{~b}$. A region of high fluorescence intensity is observed in the centre of the TEFL map indicating a high density of Brønsted acid sites at this location. A histogram of the TEFL intensity measured in the TEFL map is shown in Figure 2c. To determine the size of the most reactive zeolite region inside the measured TEFL map, we have used a global thresholding procedure described in SI section S2 to create a binary image shown in Figure $2 \mathrm{~d}$. In this image, the most reactive regions of the TEFL map are depicted in red and the TEFL intensity of these regions is marked in red in the histogram shown in Figure 2c. From the binary image, the total area of the catalytically active regions in the TEFL map is calculated to be $0.34 \mu \mathrm{m}^{2}$. For simplicity, hereafter the most reactive areas in the TEFL maps will be simply referred to as the active zeolite domains.

We note that the relative intensity of the $650 \mathrm{~nm}$ and $700 \mathrm{~nm}$ bands varies inside the TEFL map, as shown in Figure $2 \mathrm{~b}$. The thiophene oligomerization over Brønsted acid sites is known to produce fluorescent species with different numbers of monomer units. ${ }^{[18]}$ In particular, the limited availability of the Brønsted acid sites in zeolite domains has been shown to induce further oligomerization of intermediate species leading to more conjugated carbocationic oligomers. ${ }^{[16 d]}$ Furthermore, the emission wavelength of carbocationic species is positively correlated with their length and conjugation. ${ }^{[16 d, 20]}$ In other words, the $650 \mathrm{~nm}$ emission is more heavily associated with shorter oligomers compared to the $700 \mathrm{~nm}$ emission. From this we can deduce that the areas in the TEFL map exhibiting a relatively higher intensity of the $650 \mathrm{~nm}$ band compared to the $700 \mathrm{~nm}$ band must contain a relatively higher Brønsted acid site density. Figure 2e shows the profile of the average intensity ratio of the $650 \mathrm{~nm}$ and $700 \mathrm{~nm}$ TEFL bands $\left(\mathrm{I}_{650} / \mathrm{I}_{700}\right)$ measured in the regions marked in Figure $2 \mathrm{~d}$ along the direction of the arrow. In this plot, $I_{650} / I_{700}$ shows an increasing trend from outside the zeolite domain to the centre. This trend is also directly evident from the average TEFL spectra plotted in $2 \mathrm{f}$ from locations $\mathrm{A}-\mathrm{C}$ marked in Figure $2 \mathrm{~b}$, in which a higher $\mathrm{I}_{650} / \mathrm{I}_{700}$ is observed in the central region of the zeolite domain at location $A$ compared to the outer regions at locations $B$ and C. This implies that shorter conjugated carbocationic species are produced in the central part of the zeolite domain compared to the peripheral part, indicating that the central part of the zeolite domain contains a higher density of Brønsted acid sites and fewer mesopores. As more conjugated thiophene oligomers are formed in the outer regions of the zeolite domain this also implies that there is more space available to accommodate these fluorescent reaction products; hence, it can be concluded that there is a mesoporosity gradient present from outer to inner regions of the zeolite domains.
For comparison, we have also carried out TEFL mapping in regions (Figure 2a) located off-centre (locations 2 and 4) and at the edge (locations 3 and 5) of the FCC particle. The TEFL maps measured at these locations are presented in Figures 3a-3d along with the corresponding histograms of their TEFL intensity (Figures $3 \mathrm{e}-3 \mathrm{~h}$ ). In these TEFL maps, the reactive regions were identified using the same global thresholding procedure as before and are presented as binary images in Figures $3 \mathrm{i}-3 \mathrm{I}$. Average intensity profiles of $\mathrm{I}_{650} / \mathrm{I}_{700}$ in the regions marked in Figures $3 \mathbf{i}-3 \mathbf{l}$ along the direction of the arrow are shown in Figures $3 \mathrm{~m}-3 \mathrm{p}$. Interestingly, the $\mathrm{I}_{650} / \mathrm{I}_{700}$ ratio shows a general increasing trend from outside of the zeolite domains towards the centre in all four TEFL maps. This indicates that the distribution of the Brønsted acidity within zeolite domains follows a similar trend throughout the FCC particle irrespective of the location: the outer part of the zeolite domains contains fewer Brønsted acid sites and more mesopores than the central part. To obtain statistical insights into the distribution of active zeolite domains and evaluate the reproducibility of our results, we performed similar TEFL measurements at different locations (centre, off-centre and edge regions) in the two additional FCC particles (i.e., FCC2 and FCC3). The results for FCC2 and FCC3 are presented in Figures S6 and S7, respectively. All five TEFL maps in each FCC particle were measured using the same Agcoated TEFL probe and the TEFL measurements of all three FCC particles were performed using the TEFL probes prepared in the same batch. Following the same procedure used for FCC1 (Figures 2 and 3), a similar $I_{650} / I_{700}$ ratio trend was observed for the zeolite domains irrespective of their location inside the FCC particles.

Based on the TEFL microscopy results, we compared intraand inter-particle differences in the size of active zeolite domains. In the calculation of domain size, the areas $<0.002 \mu \mathrm{m}^{2}$ (corresponding to $<5$ pixels), meeting the thresholding criterion were ignored. Histograms of the areas of active zeolite domains measured from the TEFL maps in the three FCC particles are presented in Figure $\mathrm{S} 9$ based on the analysis of 16 , 17 and 14 active zeolite domains, respectively. Although $>75 \%$ of the active domains in all three FCC particles have areas $<0.04 \mu \mathrm{m}^{2}$ (corresponding spherical particle diameter: $127 \mathrm{~nm}$ ), a significant variation in the zeolite domain size could be observed within individual FCC particles. The average areas of active zeolite domains in the three FCC particles are plotted in Figure 4a. Unlike the large intra-particle variation, the average areas of the active zeolite domains measured in the three FCC particles are found to be rather similar. Figure $4 b$ shows the histogram of the areas of 47 active zeolite domains measured in all three FCC particles. $64 \%$ of the active zeolite domains have an area $<0.02 \mu \mathrm{m}^{2}$, which corresponds to a spherical particle of diameter $80 \mathrm{~nm}$. This is smaller than the previously reported values of zeolite domain size measured in similar FCC particles using CFM $(\approx 500 \mathrm{~nm}){ }^{[16 c]}$ Super-resolution Optical Fluctuation Imaging (SOFI) $(\approx 500 \mathrm{~nm})^{[17 \mathrm{a}]}$ and Scanning Transmission X-ray Microscopy (STXM) $(\approx 400 \mathrm{~nm}) .^{[21]}$ See SI section S3 for a discussion of the spatial resolution of TEFL measurements. These results show that the high spatial resolution as well as surface sensitivity offered by TEFL can successfully 

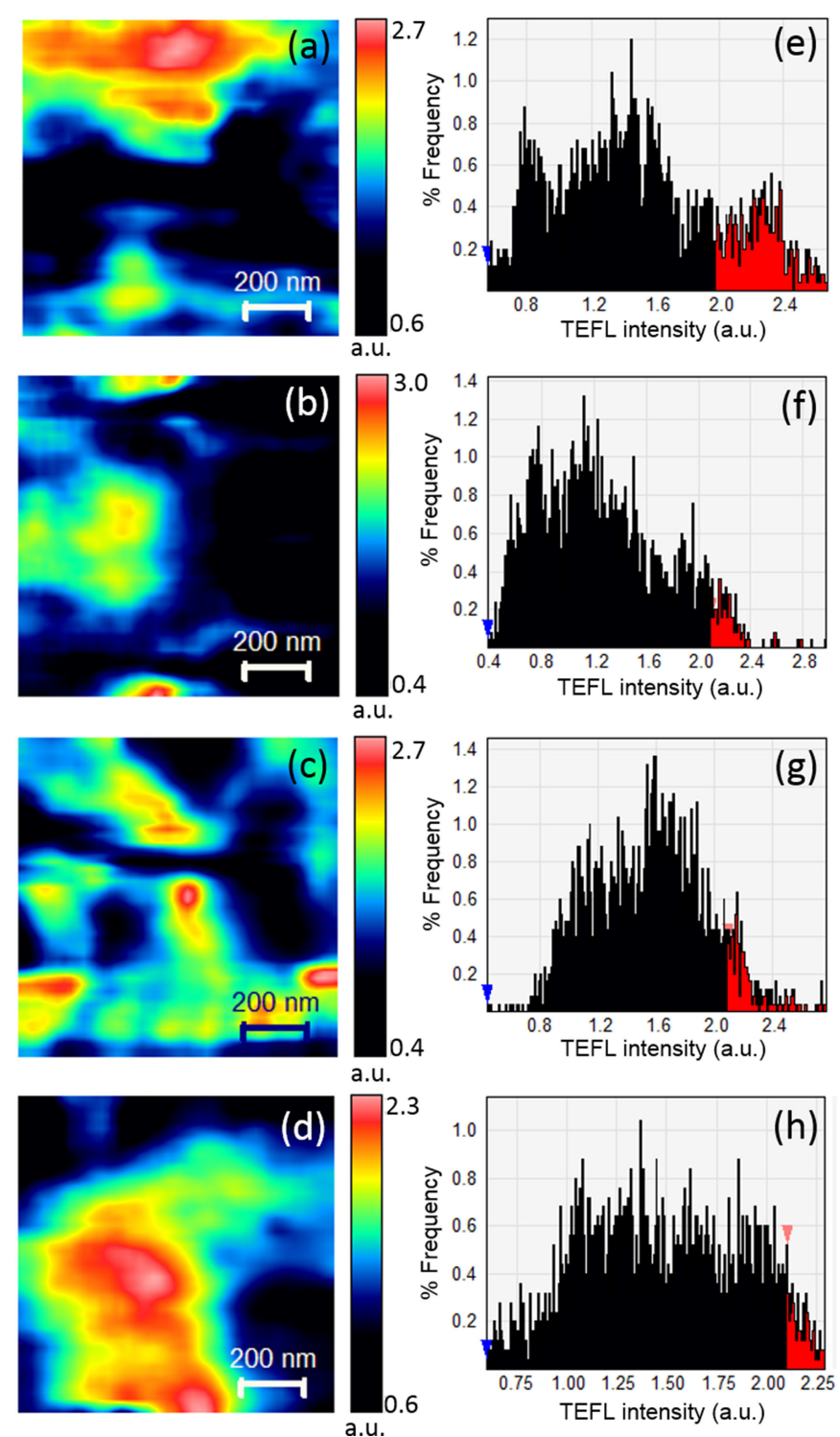
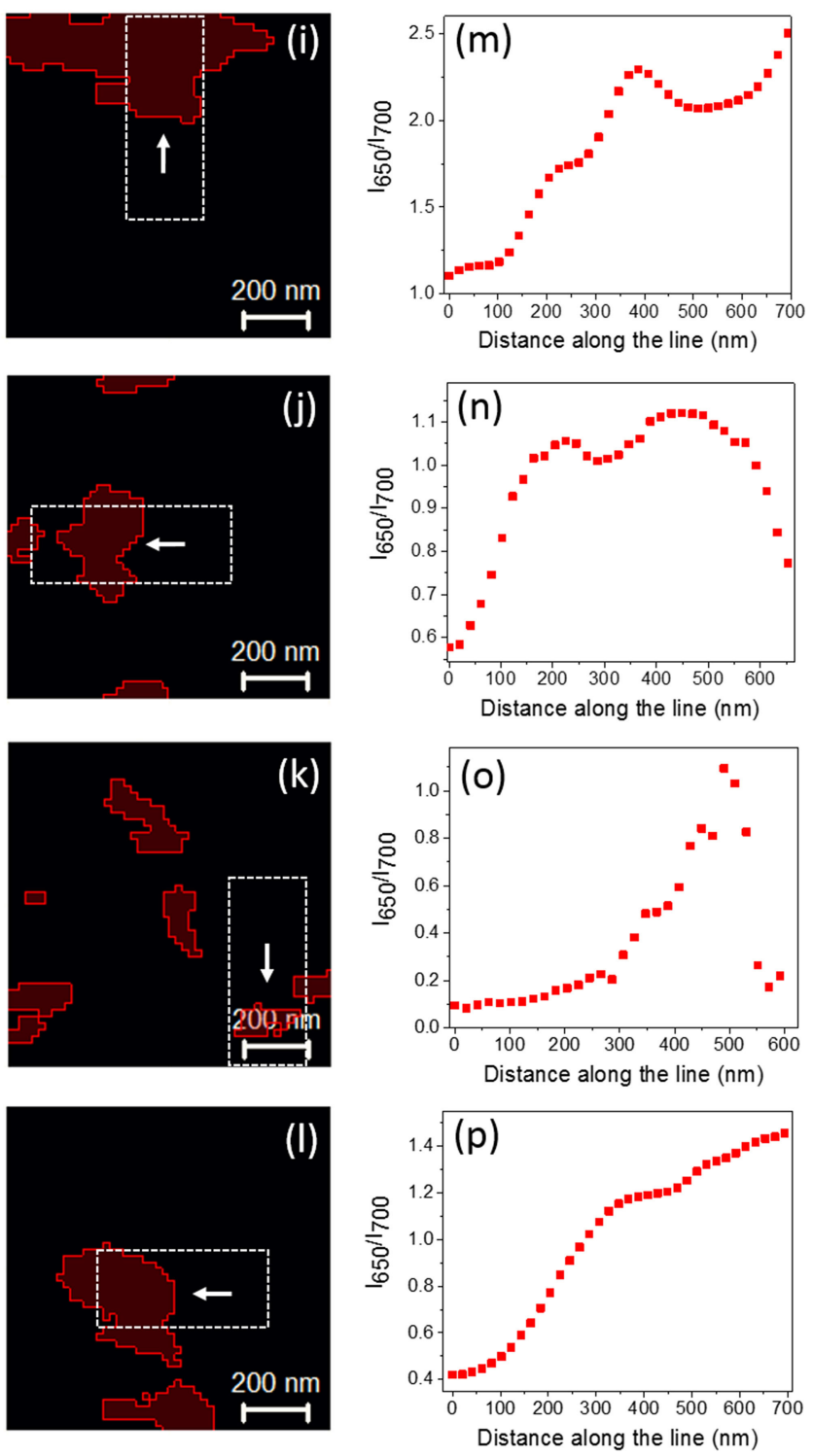

Figure 3. a)-d) TEFL maps measured from $1 \times 1 \mu \mathrm{m}^{2}$ areas marked as 2-5 inside the FCC particle (FCC1) shown in Figure $2 a$ Nominal pixel size: $20 \mathrm{~nm}$. Integration time: $1 \mathrm{~s}$. Laser power: $365 \mu \mathrm{W}$. e)-h) Histograms of the TEFL intensity in the maps shown in Figures 3a-3d, respectively. i)-I) Binary images of the active zeolite domains in the TEFL maps shown in Figures $3 a-3 \mathrm{~d} . \mathrm{m})-\mathrm{p}$ ) Average intensity profiles of ${ }_{650} / \mathrm{I}_{700}$ in the regions marked in Figures $3 \mathrm{i}-3 \mathrm{I}$ along the direction of the arrow.

identify, localize the evaluate acidity and reactivity within single zeolite domains in a FCC particle.

An inter-particle comparison of the acidity of 47 zeolite domains estimated from their average TEFL intensity is presented in Figure 4c. Unlike zeolite domain size, a significant variation in the activity is observed across the three FCC particles. FCC1 shows a significantly lower average zeolite activity (average TEFL intensity: $2.3 \pm 0.1$ a.u.) compared to FCC2 (average TEFL intensity: $3.6 \pm 0.3$ a.u.) and FCC3 (average TEFL intensity: $5.9 \pm 0.5$ a.u.). This indicates that the Brønsted acidity varies significantly within the zeolite domains for the three FCC particles and is not necessarily related to the zeolite domain size. This is further substantiated by the plot of the areas of active zeolite domains versus their average TEFL intensity, as shown in Figure S10. This plot shows that the average TEFL intensity within the active zeolite domains is independent of their size. A histogram of the total TEFL intensity measured in all 47 zeolite domains across three FCC particles is presented in Figure 4d. The histogram reveals that $10 \%$ of the most active zeolite domains have $3 \times$ higher TEFL intensity $(6.4 \pm 0.3$ a.u.) compared to $10 \%$ of the least active zeolite domains $(2.1 \pm 0.1$ a.u.), which points towards a significant inhomogeneity in the Brønsted acidity within these industrially used FCC particles. The areas of all active zeolite domains and their average TEFL activity measured in FCC particles 1-3 are summarised in Tables S1-S3, respectively.

Finally, we have studied the variation in the areas of active zeolite domains with respect to their position within the FCC particles. Figure $4 \mathrm{e}$ shows a plot of the average area of active zeolite domains with respect to three different regions within 

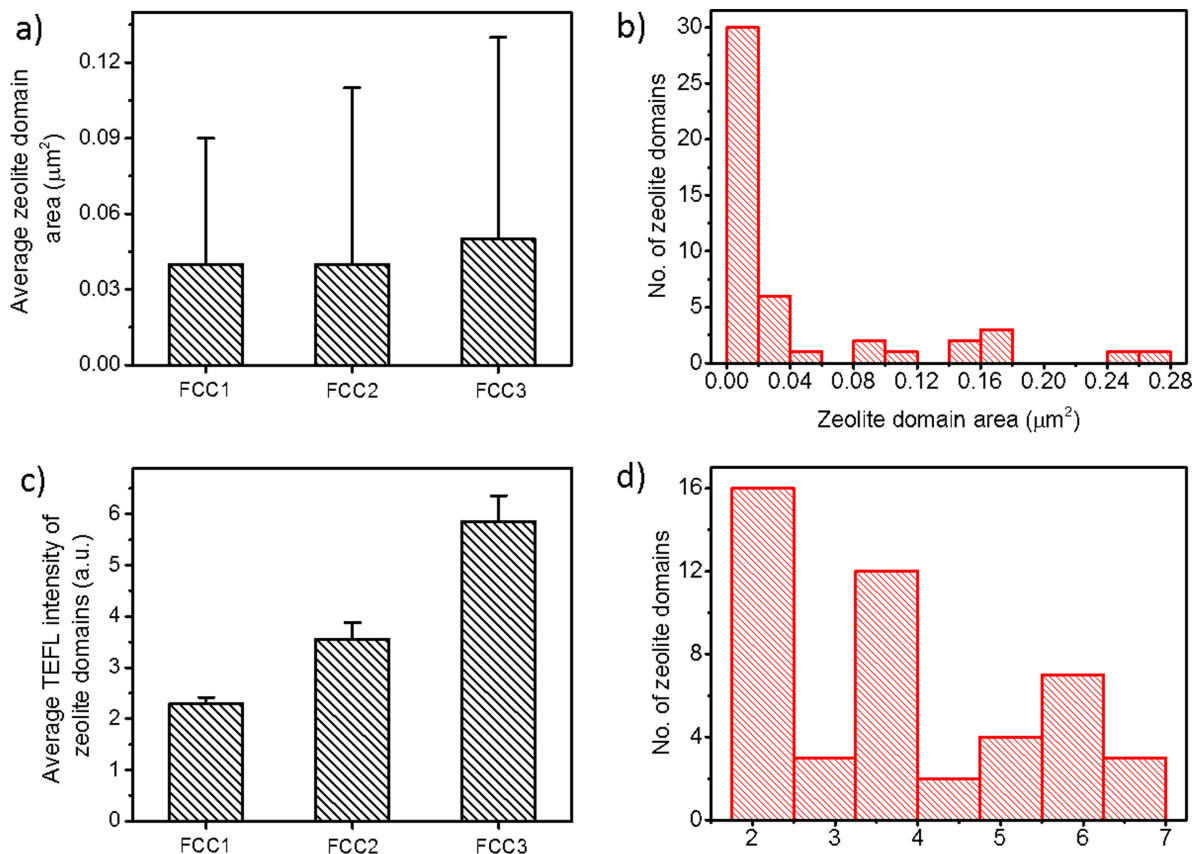

d)
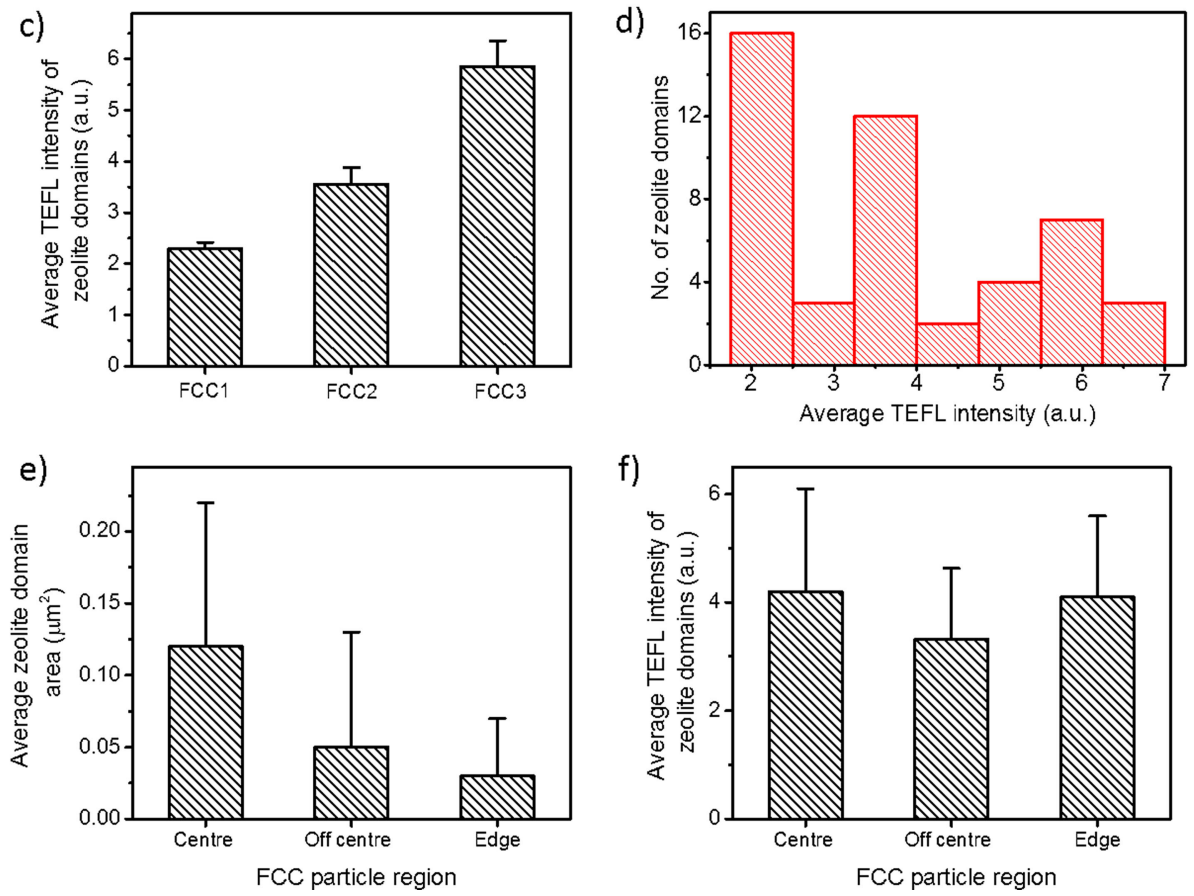

Figure 4. a) Average areas of active zeolite domains measured using TEFL microscopy within FCC particles 1-3. b) Histogram of the areas of 47 active zeolite domains measured within the three FCC particles. c) Average TEFL intensity measured within the active zeolite domains in FCC particles 1-3. d) Histogram of the average TEFL intensity measured inside the 47 active zeolite domains within the three FCC particles (i.e., FCC1, FCC2 and FCC3). Error bars in parts (a) and (c) represent the sample standard deviation. Plots showing (e) the average area of active zeolite domains and (f) the average TEFL intensity of active zeolite domains with respect to the centre, off centre and edge regions of the three FCC particles.

FCC particles: centre, off-centre and edge. This plot reveals that the active zeolite domains located in the centre of the three FCC particles have a larger average size compared to the offcentre and edge regions. By contrast, the average TEFL intensity of the active zeolite domains is found to be largely independent of their location within the FCC particles (Figure 4f). This suggests that zeolite deactivation within the FCC particles under study is not limited by mass transfer limitations or overall particle accessibility.

In conclusion, we have presented the first application of TEFL microscopy in the field of catalysis. This has been accomplished by probing the acidity and reactivity of individual zeolite domains using a selective staining reaction within a catalytic cracking catalyst particle. The hyperspectral information gained from the TEFL maps revealed a spatial distribution of Brønsted acidity within the individual zeolite domains in different regions of the catalyst particle. We have also shown that sectioning of catalyst particles into thin layers is an effective strategy to investigate zeolite acidity in the central regions of the spherical particles, which are not easily accessible by super-resolution fluorescence microscopy. ${ }^{[17 a]}$ These results demonstrate that TEFL can become a powerful and sensitive characterization tool to investigate single catalyst particles and reveal inter- and intra-particle chemical variations with nanoscale spatial resolution. Such detailed insights into the variation in catalytic activity at nanometre length scales can help understand the degradation mechanisms of FCC particles used in an industrial environment thereby facilitating the rational design of highly efficient and durable catalyst particles.

\section{Acknowledgements}

BMW acknowledges the Netherlands Organization for Scientific Research (NWO) for a Top research grant and the European Research Council (ERC) for an Advanced Grant (no. 321140). NK 
and AJW acknowledge support from International Secondment Scheme and the National Measurement System of the UK Department of Business, Energy \& Industry Strategy.

\section{Conflict of Interest}

The authors declare no conflict of interest.

Keywords: heterogeneous catalysis - fluid catalytic cracking • tip-enhanced fluorescence microscopy $\cdot$ nanoscale chemical imaging $\cdot$ acidity

[1] N. Mauser, A. Hartschuh, Chem. Soc. Rev. 2014, 43, 1248-1262.

[2] S. Kawata, V. M. Shalaev, Tip enhancement, Elsevier, Amsterdam, 2007.

[3] a) T. Deckert-Gaudig, A. Taguchi, S. Kawata, V. Deckert, Chem. Soc. Rev. 2017, 46, 4077-4110; b) P. Verma, Chem. Rev. 2017, 117, 6447-6466; c) X. Wang, S.-C. Huang, T.-X. Huang, H.-S. Su, J.-H. Zhong, Z.-C. Zeng, M.-H. Li, B. Ren, Chem. Soc. Rev. 2017; d) N. Kumar, S. Mignuzzi, W. Su, D. Roy, EPJ Tech. Instrum. 2015, 2, 9.

[4] S. Najjar, D. Talaga, L. Schue, Y. Coffinier, S. Szunerits, R. Boukherroub, L. Servant, V. Rodriguez, S. Bonhommeau, J. Phys. Chem. C 2014, 118, 1174-1181.

[5] S. Berweger, C. C. Neacsu, Y. Mao, H. Zhou, S. S. Wong, M. B. Raschke, Nat. Nanotechnol. 2009, 4, 496-499.

[6] L. Xue, W. Li, G. G. Hoffmann, J. G. P. Goossens, J. Loos, G. de With, Macromolecules 2011, 44, 2852-2858.

[7] X. Wang, D. Zhang, K. Braun, H. J. Egelhaaf, C. J. Brabec, A. J. Meixner, Adv. Funct. Mater. 2010, 20, 492-499.

[8] E. M. van Schrojenstein Lantman, T. Deckert-Gaudig, A. J. G. Mank, V. Deckert, B. M. Weckhuysen, Nat. Nanotechnol. 2012, 7, 583-586.

[9] R. Zhang, Y. Zhang, Z. C. Dong, S. Jiang, C. Zhang, L. G. Chen, L. Zhang, Y. Liao, J. Aizpurua, Y. Luo, J. L. Yang, J. G. Hou, Nature 2013, 498, 82-86.

[10] Y. Okuno, Y. Saito, S. Kawata, P. Verma, Phys. Rev. Lett. 2013, 111, 216101

[11] W. Su, D. Roy, J. Vac. Sci. Technol. B 2013, 31, 041808.

[12] A. J. Pollard, N. Kumar, A. Rae, S. Mignuzzi, W. Su, D. Roy, J. Mater Nanosci. 2014, 1, 39-49.

[13] a) W. Su, N. Kumar, S. Mignuzzi, J. Crain, D. Roy, Nanoscale 2016, 8, 10564-10569; b) N. Kumar, A. Zoladek-Lemanczyk, A. A. Guilbert, W. Su,
S. M. Tuladhar, T. Kirchartz, B. C. Schroeder, I. McCulloch, J. Nelson, D. Roy, Nanoscale 2017, 9, 2723-2731.

[14] a) F. M. Huang, F. Festy, D. Richards, Appl. Phys. Lett. 2005, 87; b) J. M. Gerton, L. A. Wade, G. A. Lessard, Z. Ma, S. R. Quake, Phys. Rev. Lett 2004, 93, 180801; c) C. Xie, C. Mu, J. R. Cox, J. M. Gerton, Appl. Phys. Lett. 2006, 89, 143117

[15] a) W. Vermeiren, J.-P. Gilson, Top. Catal. 2009, 52, 1131-1161; b) E. T. C. Vogt, B. M. Weckhuysen, Chem. Soc. Rev. 2015, 44, 7342-7370.

[16] a) I. L. C. Buurmans, J. Ruiz-Martínez, W. V. Knowles, D. van der Beek J. A. Bergwerff, E. T. Vogt, B. M. Weckhuysen, Nat. Chem. 2011, 3, 862867 ; b) I. L. C. Buurmans, J. Ruiz-Martínez, S. L. van Leeuwen, D. van der Beek, J. A. Bergwerff, W. V. Knowles, E. T. C. Vogt, B. M. Weckhuysen, Chem. Eur. J. 2012, 18, 1094-1101; c) C. Sprung, B. M. Weckhuysen, Chem. Eur. J. 2014, 20, 3667-3677; d) G. T. Whiting, F. Meirer, D. Valencia, M. M. Mertens, A.-J. Bons, B. M. Weiss, P. A. Stevens, E. De Smit, B. M. Weckhuysen, Phys. Chem. Chem. Phys. 2014, 16, 21531-21542.

[17] a) Z. Ristanović, M. M. Kerssens, A. V. Kubarev, F. C. Hendriks, P. Dedecker, J. Hofkens, M. B. Roeffaers, B. M. Weckhuysen, Angew. Chem. Int. Ed. 2015, 54, 1836-1840; Angew. Chem. 2015, 127, 1856-1860; b) F. C. Hendriks, F. Meirer, A. V. Kubarev, Z. Ristanović, M. B. Roeffaers, E. T. C. Vogt, P. C. Bruijnincx, B. M. Weckhuysen, J. Am. Chem. Soc. 2017, 139, 13632-13635; c) F. C. Hendriks, S. Mohammadian, Z. Ristanović, S. Kalirai, F. Meirer, E. T. C. Vogt, P. C. A. Bruijnincx, H. C. Gerritsen, B. M. Weckhuysen, Angew. Chem. Int. Ed. 2018, 57, 257-261; Angew. Chem. 2018, 130, 263-267; d) J. Van Loon, K. P. Janssen, T. Franklin, A. V. Kubarev, J. A. Steele, E. Debroye, E. Breynaert, J. A. Martens, M. B. J. Roeffaers, ACS Catal. 2017, 7, 5234-5242.

[18] S. C. Rasmussen, S. J. Evenson, C. B. McCausland, Chem. Commun. 2015 $51,4528-4543$.

[19] N. Kumar, A. Rae, D. Roy, Appl. Phys. Lett. 2014, 104, 123106.

[20] M. H. F. Kox, A. Mijovilovich, J. J. H. B. Sättler, E. Stavitski, B. M. Weckhuysen, ChemCatChem 2010, 2, 564-571.

[21] S. Kalirai, P. P. Paalanen, J. Wang, F. Meirer, B. M. Weckhuysen, Angew. Chem. 2016, 128, 11300-11304; Angew. Chem. Int. Ed. 2016, 55, 1113411138.

Manuscript received: June 26, 2018

Accepted Article published: July 11, 2018

Version of record online: July 31, 2018 